${ }_{\text {la revue }}^{+}+{ }^{+}$La revue pour l'histoire du CNRS

POUR LHSTSORE DU CNRS $\quad 23 \mid 2008$

Astrophysique : une science en expansion

\title{
IHÉS, la preuve par 50
}

Jean-Pierre Bourguignon

\section{(2) OpenEdition}

Journals

Édition électronique

URL : https://journals.openedition.org/histoire-cnrs/8983

DOI : 10.4000/histoire-cnrs.8983

ISSN : 1955-2408

Éditeur

CNRS Éditions

Édition imprimée

Date de publication : 31 décembre 2008

ISBN : 978-2-271-06695-4

ISSN : 1298-9800

Référence électronique

Jean-Pierre Bourguignon, «IHÉS, la preuve par 50 », La revue pour l'histoire du CNRS [En ligne], 23 |

2008, mis en ligne le 03 janvier 2011, consulté le 20 mai 2021. URL : http://journals.openedition.org/ histoire-cnrs/8983 ; DOI : https://doi.org/10.4000/histoire-cnrs.8983

Ce document a été généré automatiquement le 20 mai 2021.

Comité pour l'histoire du CNRS 


\title{
IHÉS, la preuve par 50
}

\author{
Jean-Pierre Bourguignon
}

$1 \mathrm{Au}$ lendemain de la seconde guerre mondiale, la fuite de nombre de savants allemands fait craindre une dépendance économique mais aussi scientifique des pays de la vieille Europe vis-à-vis d'Uncle Sam. Fort de ce constat, Léon Motchane ${ }^{1}$, un industriel féru de mathématiques d'origine russe mais vivant en France depuis une vingtaine d'années, affiche alors une ambition plutôt " révolutionnaire » : offrir à des savants d'envergure exceptionnelle un lieu consacré à la recherche, sans aucune obligation d'enseignement ou de tâches administratives, pouvant aussi accueillir des visiteurs temporaires. L'idée d'un centre en gestion privée au service de la création scientifique, vivante, polymorphe et évolutive prend forme.

2 Léon Motchane a l'intuition de l'importance de recréer les conditions pour une interaction intense entre mathématiciens et physiciens. Il cherche des soutiens pour son projet et noue des relations avec des scientifiques de plusieurs pays, dont des Américains. Sa passion pour les sciences et la recherche va le conduire à créer l'IHÉS. "Il fallait un lieu où les choses fussent organisées pour que les gens aient la plus grande liberté intellectuelle ", explique Didier Motchane, l'un de ses fils. C'est ainsi qu'avec le soutien de Jean Dieudonné et de Robert Oppenheimer, alors directeur de l'IAS, naît l'association "Institut des hautes études scientifiques». Le centre fonctionne de façon assez informelle au début. Les premiers fonds, très modestes, nécessaires à sa fondation et à ses premiers pas, furent fournis par quelques grandes sociétés industrielles, dont les présidents étaient, pour certains, d'anciens ingénieurs. L'État français commencera à donner un soutien à partir de 1965, à la suite d'une décision du Georges Pompidou, alors Premier ministre.

3 Hébergée pendant les trois premières années de son existence à la Fondation Thiers, à Paris, l'association déménage ensuite en 1962 à Bures-sur-Yvette dans le domaine de Bois-Marie, qu'elle occupe toujours. L'association est reconnue d'utilité publique depuis 1961. La première aile du bâtiment scientifique est construite en 1963, deux autres, édifiées en 1973 et 1983, complètent le bâtiment scientifique qui prendra sa forme actuelle après la reconstruction de la première aile en 2001 et la rénovation des deux autres ailes en 2004. La résidence de l'Ormaille, composée de 37 studios et pavillons, est 
construite en 1967. En 1998, après l'avoir louée pendant 31 ans, l'IHÉS parvient à l'acquérir en contractant un emprunt de 25 MF sur 25 ans. Depuis les années 1970, de grands organismes de recherche européens, comme l'Engineering and Physical Sciences Research Council, la Max-Planck Gesellschaft, la Société helvétique des sciences naturelles et les services du Premier ministre belge contribuent au fonctionnement de l'Institut.

Depuis 1985, la National Science Foundation des États-Unis lui apporte aussi une contribution à laquelle s'ajoute depuis une dizaine d'années celle de fondations japonaise et chinoise.

«Art. 1 : La fondation dite « institut des hautes études scientifiques» a pour but de favoriser et de faire effectuer des recherches scientifiques théoriques dans les domaines suivants : mathématiques, physique théorique, méthodologie des sciences de l'homme, et de toute discipline théorique qui entretient des liens avec elle.»

Une émulation intellectuelle et un brassage permanents

L'IHÉS recrute ses professeurs permanents jeunes (moins de 40 ans) en faisant un pari fondé sur l'envergure et la profondeur de leurs travaux au moment de leur recrutement ${ }^{2}$. Le CNRS participe à cet effort en mettant à la disposition de l'Institut quelques chercheurs sélectionnés aussi rigoureusement comme visiteurs de longue durée. Le talent et la créativité des personnalités scientifiques exceptionnelles qui ont fait l'histoire de l'IHÉS ont ouvert la voie à des manières de penser originales et novatrices et donné naissance à des concepts inédits dans l'approche des mathématiques et de la physique théorique. Les chercheurs de l'Institut y travaillent en toute liberté (de recherche et de méthodes de travail) et font vivre l'interactivité entre les différentes disciplines qui y sont représentées (mathématiques, physique, biologie, informatique...).

Au Panthéon des mathématiques et de la physique

6 La recherche fondamentale, objet des travaux des scientifiques de l'IHÉS, cherche à repousser sans cesse les limites du savoir. Elle permet de jeter les bases de nouvelles branches et de nouveaux concepts dont l'étendue ne peut être déterminée qu'a posteriori et dont les paradigmes principaux se révèlent parfois pertinents dans des secteurs pour le moins inattendus et loin des domaines qui les ont motivés initialement. Un bel exemple en est donné par la «théorie déterministe du chaos ${ }^{3}$ dont les bases ont été posées à l'IHÉS.

7 L'histoire de l'IHÉS a été marquée par des personnalités scientifiques hors du commun qui ont contribué, chacune à sa façon mais de manière décisive, à l'évolution des sciences mathématiques et physiques au cours du XXe siècle. Ils ont fait de l'Institut un point de référence dans leur spécialité. C'est ainsi que sept des neuf professeurs permanents de l'Institut, mathématiciens, ont reçu la médaille Fields ${ }^{4}$, la plus haute distinction au niveau mondial en mathématiques.

8 Alexander Grothendieck, premier professeur permanent de l'Institut, a créé le nouveau cadre de pensée de la géométrie algébrique moderne, cadre qui est maintenant universellement adopté. Son travail a encore été amplifié par Pierre Deligne, à l'origine son disciple, recruté à l'IHÉS à 26 ans et maintenant installé à l'IAS. Ce dernier a considérablement contribué à l'émergence de ce qu'on appelle aujourd'hui la "géométrie arithmétique»: cette approche très particulière des propriétés des nombres entiers à laquelle on peut aussi rattacher André Weil (frère de la philosophe Simone Weil) a été le soubassement de la preuve du théorème de Fermat, ouvert pendant plus de trois siècles. 
9 Le physicien Louis Michel et le mathématicien René Thom ont rejoint l'IHÉS respectivement en 1962 et 1963, suivis par un autre physicien, David Ruelle, en 1964. Louis Michel a été, comme Eugene Wigner, l'un des grands promoteurs de l'usage de la théorie des groupes en physique, tant dans la théorie des particules élémentaires qu'en cristallographie. René Thom a couvert un champ immense : partant de la topologie, cette branche des mathématiques qui s'intéresse aux formes à déformation près, il a développé une "mathématique de la morphogenèse ", proposant des modèles pour la biologie et aussi pour les sciences humaines. Ces propositions, souvent regroupées sous le nom de "théorie des catastrophes ", ont quelquefois été controversées, mais ont fait l'objet d'un intérêt considérable hors de la communauté scientifique. Il a consacré la suite de sa vie scientifique à l'étude de la biologie théorique et surtout de la philosophie aristotélicienne. David Ruelle a été le premier promoteur des modèles déterministes de la turbulence, son travail est englobé aujourd'hui dans la théorie moderne du chaos.

10 Dennis Sullivan, professeur permanent de 1974 à 1997, mathématicien américain, a fait alterner, pendant des années, ses activités de recherche entre les États-Unis et la France. Topologue, spécialiste de dynamique, il avait un talent incomparable pour favoriser les échanges entre les visiteurs et les motiver pour aller au plus profond de leurs idées. Jürg Fröhlich et Oscar Lanford III, tous deux physiciens théoriciens américains, ont été professeurs permanents respectivement de 1978 à 1983 et de 1982 à 1987 avant de s'installer définitivement à l'ETH (Eidgenössische Technische Hochschule) à Zürich. Jean Bourgain, mathématicien belge spécialiste d'analyse sous beaucoup de formes, a rejoint l'Institut en 1984 puis l'IAS en 1994. Il a reçu la médaille Fields en 1994.

11 «Le siècle qui commence s'annonce comme celui où économie et nouvelles technologies se conjuguent étroitement. l'institut des hautes études scientifiques, qui s'est forgé une réputation exceptionnelle à la pointe des sciences théoriques et des mathématiques, a donc un rôle exceptionnel à jouer dans l'avenir de l'Europe comme dans celui du monde. » Raymond Barre

Ouverture à l'international

12 Depuis sa création, l'IHÉS a reçu plus de 5000 chercheurs provenant de plus de 60 pays. Il accueille chaque année environ 250 visiteurs avec en moyenne une cinquantaine de chercheurs simultanément sur le site. Depuis 2004, l'IHÉS soutient, en partenariat avec la fondation Schlumberger, le développement de la science fondamentale en Afrique sub-saharienne en subventionnant des formations de haut niveau de chercheurs et étudiants et en prenant entièrement en charge les visites de chercheurs en provenance de ces pays. Par ailleurs, l'Institut travaille activement à étendre son action et sa visibilité sur le continent asiatique, avec dans un premier temps, des efforts déterminés en direction du Japon et de la Chine.

Toujours l'interdisciplinarité et des ponts vers l'industrie

13 L'Institut doit également son attractivité à sa localisation dans le bassin parisien, où se trouve la plus grande concentration de mathématiciens au monde, ainsi qu'à sa proximité de centres d'excellence tels que le centre d'Orsay de l'université Paris-Sud, l'École polytechnique, le site de Saclay du Commissariat à l'énergie atomique, le campus de Gif-sur-Yvette du CNRS ou des centres de recherche de grandes entreprises comme Danone ou Thalès.

14 Le champ d'exploration a été élargi en lançant, en 2001, de nouvelles activités à l'interface des sciences mathématiques et de la biologie moléculaire. L'exploration de problèmes fondamentaux trouvant leur origine dans un cadre industriel viendra 
compléter cette ouverture. Développer cette nouvelle frontière apparaît aujourd'hui au coeur de l'essor de nouvelles technologies mais c'est l'impact de ces questionnements sur la recherche fondamentale qui intéresse l'IHÉs.

\section{NOTES}

1. Né en 1900 à Saint-Pétersbourg, Léon Motchane émigre en Suisse en 1918 dans des conditions très pénibles puis s'installe définitivement en France en 1924. C'est à l'âge de 53 ans qu'il reprend ses études en mathématiques interrompues dans sa jeunesse et soutient sa thèse sous la direction de Gustave Choquet.

2. Les deux derniers mathématiciens recrutés comme professeurs permanents par l'IHÉS (Maxim Kontsevitch en 1995 et Laurent Lafforgue en 2000) ont tous deux reçu la médaille Fields, respectivement en 1998 et 2002.

3. Raccourci consacré pour désigner l'évolution des systèmes déterministes qui sont très sensibles aux variations des conditions initiales.

4. Parmi les 48 lauréats, 45 ont séjourné à l'IHÉS.

\section{RÉSUMÉS}

L'Institut des hautes études scientifiques (IHÉS) de Bures-sur-Yvette est un institut de recherche avancée en mathématiques et physique théorique avec, depuis 2000, une ouverture sur l'interface mathématiques-biologie et médecine. Conçu comme le pendant européen du célèbre Institute for Advanced Study (IAS) de Princeton, l'IHÉS a fêté ses 50 ans en 2008.

The Institut des hautes études scientifiques (IHÉS) is an institute of advanced research in mathematics and theoretical physics that has developed, since 2000, new activities at the interface betweeen mathematics and biology and medicine. The IHÉS celebrated its 50th anniversary in 2008.

\section{INDEX}

Mots-clés : physique, mathématiques, Institut des hautes études scientifiques, IHÉS, médaille Fields 


\section{AUTEUR}

\section{JEAN-PIERRE BOURGUIGNON}

Géomètre différentiel de formation, Jean-Pierre Bourguignon s'est ensuite intéressé aux aspects mathématiques des théories physiques, de la théorie de Yang-Mills à la relativité générale. Directeur de recherche au CNRS et enseignant à l'École polytechnique, il a présidé la Société mathématique de France et la Société mathématique européenne. Il dirige l'IHÉS depuis 1994. 